\title{
Sleep Time Transfer
}

\author{
Xincun Chen \\ School of Economics and Business Management, Southwest Jiaotong University, Chengdu, China \\ Email: cxc9608@163.com
}

Received March 13 ${ }^{\text {th }}$, 2013; revised April 16 ${ }^{\text {th }}$, 2013; accepted May $15^{\text {th }}, 2013$

\begin{abstract}
Copyright ( 2013 Xincun Chen. This is an open access article distributed under the Creative Commons Attribution License, which permits unrestricted use, distribution, and reproduction in any medium, provided the original work is properly cited.
\end{abstract}

\begin{abstract}
The goal of the article is to definite that sleep with sufficient time and depth is a high quality sleep regardless of whether the sleep time changes or not. The author had conducted an experiment about sleep time. During the experiment, the author changed participants' main sleep time four times. The result of the survey shows that participants take a few days to adapt to the new condition after their main sleep times have changed. It would not affect the recovery of the energy of the people even though the time point of their main sleep changes.
\end{abstract}

Keywords: Sleep Time; Transfer

\section{Introduction}

Sleep is a kind of quiescent condition which is spontaneous and reversible. It appears periodically among higher vertebrates. Its symptoms are the decrease of body reactive toward External stimuli and temporarily interruption of consciousness (Fuller, Gooley, \& Saper, 2006). Sleep, which is the basis of keeping health and recovery, is a kind of initiative process. The best way of relaxing is to get a full sleep. Modern medicine generally considers that there are specialized hubs to manage sleep and arousal. Sleep is just another work pattern of brain for storing energy. Human, similar to other higher vertebrates, always alternate the two conditions-arousal and sleep (Saper, Chou, \& Scammel, 2001). Human's physical body can’t live without sleep and food.

Medical Information (1997) reported that British and American researchers had researched the sleep time of 24 healthy adults. The experimenters are managed to sleep four hours later than usual every day. It will cause a circadian rhythm disorder syndrome a few weeks later. What we got from up researched is Everyone's body has an inner clock, which is adjusted by the extent of exposure to light and darkness. It determines the time of arousal and sleep. People would be upset if its circadian rhythm was violated by sleep time (Jiang, 1986). Yet, there are also researches on three 8-hour workers. The conclusion is that the relationship between the sleep time of different teams and the quality of sleep is not obvious. But it considers that the quality of sleep relates to work, rest ambient noise, wok tension, drinking coffee at night and smoking too much (Zhang, 2008). Britain Nature reported that American scientists considered the length of people's sleep time may be partly related to inheritance after doing research on drosophila's (Shaker) mutants. Mutation of a single gene can produce a very short sleep phenotype and put it on the shaker site. But it is not clear whether the very short sleep is related to those factors in that research (He, 1997).

Thus it can be seen that the sleep research is still superficial, even murky. Many conclusions cannot be drawn from practice. Although many sleep phenomena in daily life still cannot be explained clearly, but while doing the time management experiment, the author discovers an interesting phenomenon: sleep time migratory. I discovered this phenomenon from my experiment with sleep categories; so it is important to first understand sleep categories.

\section{Sleep Categories}

From the EEG brain waves taken from sleeping people, sleep can be classified as Slow Wave Sleep and Paradoxical Sleep. During slow wave sleep, the human brain activity progresses from low activity to high activity and then a mixture of slow and high activities. Slow wave sleep occurs during the beginning of sleep and during brief waking up. Paradoxical sleep occurs repeatedly during sleep and in a very excited state. EEG shows high brain activity, like those during waking. It has very rapid eye movement (REM) and often with active dreaming. Slow wave sleep and paradoxical sleep changes throughout the night, typically alternates four to six times.

Sleep cycle. Sleep can be classified into shallow sleep, mild sleep, moderate sleep and deep sleep. Researchers consider that there are 5 - 6 cycles in people's night sleep.

The research shows that shallow sleep and mild sleep which account for $55 \%$ of the sleep time has little effect on relieving fatigue. Yet, such a deep sleep only accounts for $25 \%$ of the entire sleep time. The evaluation of sleep should depend on the quality rather than the time. Improving sleep quality finally depends on the length of time of deep sleep. From the perspective of sleep science, the "deep sleep" is the depth of the restfulness of the brain after you fall asleep. During the sleep night, you first enter the shallow sleep and then naturally go into the deep sleep and then back and forth alternating between deep and light sleep until waking up. It is possible for people to dream in shallow sleep, but never in deep sleep.

From the research of the sleep effects for human body, the 
author believes sleep can be divided into primary period and secondary sleep period. Primary sleep segment is defined as primary sleep period, and secondary sleep segment is defined as secondary sleep period. The primary sleep period bears the primary effect of sleep. The two sleep classifications are studied according to the primary sleep period. An adults' primary sleep period time is between 5 to 9 hours. Having satisfied the primary sleep period, the amount of sleep that is needed is basically reached. Secondary sleep period is the compensation of primary sleep period. When primary sleep period does not meet the requirement, the effect of secondary sleep period will become visible. In general, an adult's secondary sleep hour is less than two hours. Some may be only a few minutes. Most of the secondary sleep period is under moderate sleep. The slowwave phase is usually in period 1-2-3-2 and so on. It is easily interfered by environment. Secondary sleep is caused by lack of primary sleep and habits. The so-called "Zi Wu sleep" means sleep deeply in midnight and nap at noon. The more actual understanding is about the primary and secondary sleep rather than sleep in midnight and at noon. There must be secondary sleep between the two primary sleeps.

Study shows that only in a full resting state before the primary sleep can cerebral cortex cells play a decisive role in eliminating fatigue, recovering energy and preventing disease. It is easy for eyes to have secretions and for oral cavity to have tartar and moss dirt after awakening is because of the deep sleep and long time in primary sleep period. The primary sleep can form a relatively stable pattern: you need to go to sleep and awake at a fixed time. This kind of pattern is what people call "inner clock". If primary sleep time is enough, the natural awakening people will feel full of energy. If you are awake from the passive state before the natural awakening time, you will feel dizzy, weak and hard to open your eyes. It is not easy for eyes and oral cavity to have secretions in secondary sleep period because of the shallow sleep and short time. Secondary sleep period also has its own "inner clock". It can also have a relatively stable pattern. But it is much easier to change than primary sleep time. Many people have a habit of noontime snooze. It is necessary to sleep for a while when the time comes, even just take a nap. If you are awake from the passive state in secondary sleep condition, you won't feel dizzy, weak or hard to open your eyes. Even if you can feel that, it may be much lighter.

Sleep time migration refers to the changes in sleep period within 24 hours, which mainly refers to the changes in the primary sleep period.

\section{The Design of the Study}

\section{The Origin of the Problem}

People usually have a sleep time. When this time arrives, people will want to sleep. When the amount of sleep time forms a habit, people will naturally wake up. This kind of life habit about "sleep" and "awake" construct the inner clock. Go to bed and get up according to the fixed time. It is natural that the inner clock sometimes is very precise. Whether the inner clock can be changed or not is still subject for a future study.

When investigating students' study time in this study, the author discovered that some students sleep more at night and less in daytime. But some students are the opposite. They sleep more in daytime and sleep less at night.
Example 1: After watching the European Cup, some students feel that their sleep time changed. The students who can't stay the night originally don't doze off any more at night after watching football match. Many people's sleep time changed. They changed to sleep from morning to afternoon.

Example 2: The sleep time habit of some teachers who do experiments at night also changed after changing their rest time for a period.

Example 3: In daily life, it often can be seen that people's sleep time is not 11 pm-1 am. Night workers sleep even in daytime. The author interviewed some workers who had to work at night, such as doctors, entrance guards, night taxi drivers and so on. They were not worried about the effect of night work to sleep. What they were worried was the time wouldn't be fixed. They thought they could also sleep well in the morning for a long time.

During the interview, the night taxi drivers thought that they also could sleep for 7 - 8 hours in daytime. Due to the less night passengers, they also catch the opportunity to nod. The entrance guards said their night shifts were relatively fixed. It changes every half a month. In their points of view, it was not bad. The hospital's doctors and nurses had often "three shifts" and primary sleep time changed frequently. They didn’t hope so. Some preferred to value the graveyard shift

The author doesn't want to deny the advice that the modern medical science tells people to sleep at 11 p.m.-1 a.m. And the author also has no intention to study what time to sleep is good for human. The subject of the study is whether the primary sleep can migrate.

\section{Question Assumption}

From interviews and social reality, there are quite a number of people's primary sleep period cannot be midnight. How do they sleep? Some people's primary sleep period moved because of some special reasons. How can they adjust it back? Would the primary sleep period move? How do people control their own sleep? The author wants to find out some rules according to the experiment.

\section{The Implementation of the Study}

\section{Subject}

Subject: 18 in-school male college students become experimental volunteers. The youngest is 21 years old, and the oldest is 24 years old, the average age is 21.95 years old.

Staff: 20 in-school male college students become experimental volunteers. They are responsible for observing and recording subjects.

\section{Time}

30 days. July 15th 2012 to August 13th.

\section{Place}

Quiet dormitory. There are six students in a dormitory. There are three dormitories in total.

\section{Experimental Observation Items}

1) Subject; 
2) Age;

3) The number of yawning between the starting time of last primary sleep time to the starting time of next primary sleep time;

4) The sleep time of this primary sleep;

5) The starting time of primary sleep this time;

6) The first natural awakening time of this time;

7) The ending time of primary sleep this time;

8) The sleep time of this secondary sleep;

9) The starting time of secondary sleep this time;

10) The natural awakening time of secondary sleep this time.

\section{Experimental Procedure}

1) The total sleeping time of a subject a day is 8 hours. Primary sleep period accounts 7 hours and secondary sleep period accounts 1 hour.

2) Transferring the primary sleep time 3 hours later. It is a kind of protection that extends 3 hours of primary sleep every week. After testing extending 5 hours of primary sleep, we can discovered the subjects' emotions are easy to become impatient after changing the starting time of primary sleep beyond 5 hours many times in a month. It can be basically accepted when reducing to 3 hours.

3) Sleep schedule is as follows:

Preparing period: The forecast period was in July 15th and 16th. The primary sleep was adjusted at 0 a.m. and get up at 7 a.m. Secondary sleep started at 13 p.m. to 14 p.m.

First week (July 17th - 23rd): The primary sleep was adjusted at 3 a.m. and get up at 10 a.m. Secondary sleep started at 16 p.m. to 17 p.m.

Second week (July 24th - 30th): The primary sleep was adjusted at 6 a.m. and get up at 13 p.m. Secondary sleep started at 19 p.m. to 20 p.m.

Third week (July 31st - August 6th): The primary sleep was adjusted at 9 a.m. and get up at 16 p.m. Secondary sleep started at 22 p.m. to 23 p.m.

Fourth week (August 7th - 13th): The primary sleep was adjusted at 12 a.m. and get up at 19 p.m. Secondary sleep started at 1 a.m. to 2 a.m.

\section{Experiment Approach}

1) Experiment approach, observation approach and statistical approach. The staff were divided into groups and took turns on duty to organized experiment and collected data at experiment field. Each subject's single observation item should be recorded each day.

2) Some of the data were written by subjects themselves.

3) Statistics aspect: using 18 people's data to count in general. In the observed certain span of magnitude larger items, remove the two highest and two lowest data, and use the remaining 14 data for statistical analysis.

\section{Result}

The adjustment of two-day probationary period is very smooth. All the 18 people can do as the requirement.

Observing the average yawning numbers between the next two sleep times.

On the provisions of the next two sleep time refers to the original sleep time and sleep time when provisions. Observing the yawn numbers is actually observing the sleepy degree. The more yawn number it has, the higher the sleepy degree it will be. From the result we can see: 1) The yawning number showed a decreasing trend in the same test weeks. 2) In the 3rd week, the average number of yawn was the highest. 3) It is interesting that yawn was infectious. Sometimes we just did not yawn a long time. Once someone yawned, most of the time someone would follow. Sometimes all the people would follow one after another, as well as the stuff. 4) The yawn number was counted by the subjects themselves.

Observation of the sleep initiation time of the primary sleep.

In the previous four days of each test week, we set a compulsory sleep initiation time, but we took a flexible way in the after three days. When view from the frequency of yawns, we noticed that during the beginning few days, people were tend to take naps to a much high degree. What's more, they apt to feel sleepy near the time they regularly fall asleep. After a few days' adjustment, it appeared to come close to the time they are made to sleep in the test, but in a scatter way. As the dispersal scopewas relatively large, this study applied mode in the statistics analysis. After a few days again adjustment, then formed a new time of sleep habit. Observation found that after the fifth day, although there was no regulation to sleep time, but, most of the participants dozed near the new sleep time, at most in 26 minutes after this time point all fell asleep. Thus a migration from the original sleep habit time to a new sleep habit time of the main sleep is finished. Four weeks' tests are so. Thus it can be deduced that sleep habit time can migrate in 24 hours.

Observation of "habitual time point reaction".

During the observation of sleep status, this study found that the reaction of participants were strong when it was close to last habitual time point to get up in the first day, almost in an amplitude of 30 minutes when it was close to the original get-up time point. All participants appeared different degree of paradoxical sleep, even awakening reaction. Some repeated keel over, snoring stopped, and some woke up to drink water or go to the toilet. Some even couldn't sleep after waking for half an hour. The phenomenon appeared after the adjustment of the sleeping time, and people still get used to reacting at the originnal period of time. In this study, we called this phenomenon the habitual time point reaction. In fact, it is much more complicated than the paradoxical sleep. After the second day, this kind of phenomenon appeared directional dispersion, and was gradually close to the new regulation time point of getting up.

The observation of primary sleep awake.

The subjects were woken after sleeping 7 hours from 1st 4 th day. The subjects were woken from the passive state. Sleep was interrupted because of the effect of "habits time point reaction” which appeared in early time. If going on sleep, the subjects would soon fall into the slow wave sleep. When primary sleep reached 7 hours, almost all of the subjects could not wake up by themselves, they needed to be woken up forcibly. Subjects generally felt tired and fatigue lethargy after waking up in first two days and all day would feel dippy. Symptoms gradually reduced after the third day. Without waking up them after 5th day, and observe the naturally waking time of the subjects. It could be observed that the majority of subjects naturally woke up at magnitude of 40 minutes to the wake-up time of the first four days.

The observation of secondary sleep.

Because of the short secondary sleep time, its corresponding changes are less. It is mainly reflected in: 


\section{C. CHEN}

1) It was less and shorter to be awake during the secondary sleep; it may say that only paradoxical sleep does not have habitual response in point-in-time;

2) They must be woken up a few days before test week. When most people had slept for nearly 1 hour, sleep still sinking, they still slept soundly. There was no indication that they would be provided to wake up naturally on time;

3) Waking them up forcibly for 4 days, and then we didn't wake them up after the 5th day, observing the natural waking time under test. From the observing we can see that most subjects woke naturally around 30 minutes of the natural awake time of the first 4 days.

Results in Table 1 showed that all testee will spent 4 days(fixed sleep time 1 - 4; awake time period 1 - 4 awaken) to be formed new habitual sleep time that in both "PRIMARY SLEEP" and "SEC SLEEP", so the day 5 - 7 (awake time period) showed natural waking up (5 natural, 6 natural, 7 natural) in new time through whole test weeks(test week 1 , test week 2 , test week 3, test week 4).

\section{Discussion}

It is easy for sleep to form a relatively stable pattern. Good sleep needs a stable time. Only developing good work habits and having a stable time of work and rest could people coordinate their physiological function well. Sleep habits can be formed within a week. However, long-term stability is conducive to good health.

Table 1.

Descriptive for relationship between sleep time and awake time by tested 18 male students.

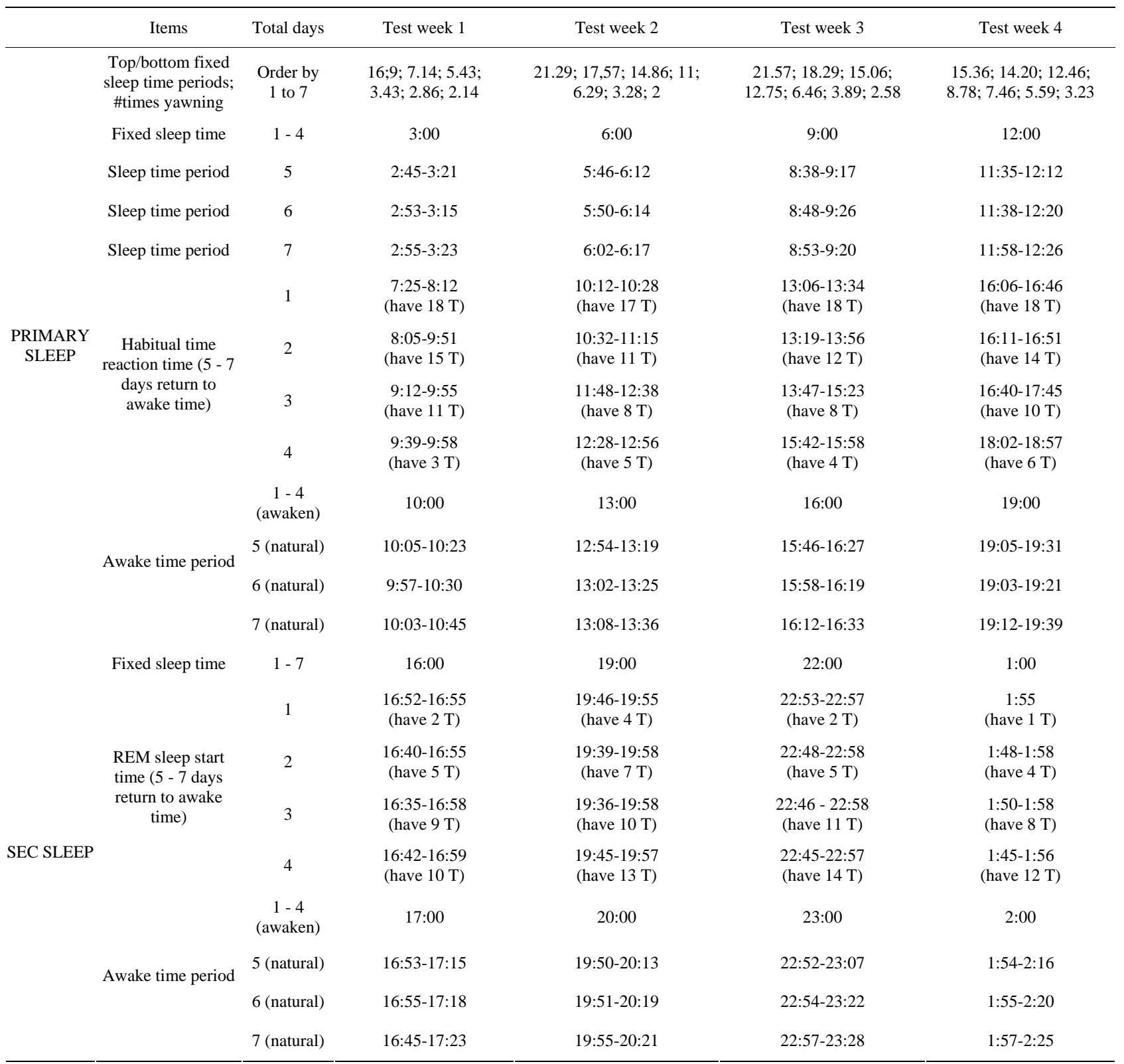

Note: $\mathrm{T}=$ Testee. SEC SLEEP = secondary sleep. 


\section{C. CHEN}

Sleep time can be transferred. Sleep experiment shows that sleep habits can be moved within 24 hours. It is customary that people can get good sleep only at night. Sleep experiments shows that the key to good sleep is not the time, day or night, but the quality of sleep. The quality of sleep includes sleep time and sleep depth. As long as the primary sleep can get the sleep time with sufficient depth, people can fully recover. Although the sleep time can be transferred, it will affect the quality of sleep if the sleep time is changed frequently. A basic condition for good quality of sleep is a regular daily routine. Therefore, one does not have to worry about he won't sleep well after midnight or night work. As long as there is a relatively fixed timetable, he also can get a good sleep.

Sleep time can be artificially controlled. According to different types and needs, you can adjust the sleep time. As long as the arrangements are proper, it will not affect the body.

Yawning is infectious. It can also be considered that sleepy is infectious. Yawning is a manifestation of exhaustion. A person's yawn can bring other people's yawning. Similarly, a per- son showing sleepy also might make other people become sleepy. Therefore, the sleepy people who are in the same group should be separated as soon as possible. Otherwise it will affect morale.

\section{REFERENCES}

Fuller, P. M., Gooley, J. J., \& Saper, C. B. (2006). Neurobiology of the sleep-wake cycle: Sleep architecture, circadian regulation and regulatory feedback. Journal of Biological Rhythms, 6, 482-493.

He, G. (1997). The physical and psychological functioning and ergonomic. Chinese Journal of Ergonomics, 2, 53-55.

Jiang, D. F. (1986). Sleep and brain function. Environmental Vibration Limit of High-Speed Railway, 2, 56-58.

Saper, C. B., Chou, T. C., \& Scammell, T. E. (2001). The sleep switch: Hypothalamic control of sleep and wakefulness. Trends Neuroscience, 12, 726-731. doi:10.1016/S0166-2236(00)02002-6

Zhang, D. (2008). The relation between sleep time and sleep quality in night time job. Science in China, 10, 78-98. 